\title{
Acerca de computación y sus líneas de investigación
}

\section{About computing and its research lines}

\author{
Rosa Delgadillo*, Armando Fermín-Pérez \\ ${ }^{1}$ Universidad Nacional Mayor de San Marcos, Facultad de Ingeniería de Sistemas e Informatica. Lima, Perú. \\ Email Rosa Delgadillo: rdelgadilloa@unmsm.edu.pe \\ Email Armando Fermín-Pérez: fferminp@unmsm.edu.pe \\ * Autor para correspondencia
}

\section{Introdución}

Las tecnologías de la información y comunicaciones se encuentran en cada actividad humana y en todos los campos de la ciencia, ingeniería, negocios entre otros, tanto así que la computación se ha convertido en un conocimiento básico que todos deben conocer. De manera general, la Association for Computing Machinery (ACM) [1] define a la computación como cualquier actividad relacionada con las computadoras y orientada a objetivos; por lo tanto, la computación incluye al diseńo y construcción de sistemas de hardware y software para una amplia gama de propósitos, relacionadas con el uso de diferentes tipos de información, la realización de estudios científicos, implementación de sistemas con comportamiento inteligente, las comunicaciones y el entretenimiento, entre muchas otras, haciendo que la lista sea prácticamente infinita y con enormes posibilidades dependiendo del contexto involucrado. Así, se hace necesario que los profesionales de la computación sean buenos en sus actividades, las que pueden llegar a ser bastante complicadas y difíciles, por lo tanto la computación no es solo una profesión sino también una disciplina, o una familia de disciplinas relacionadas con todos los campos del saber y el conocimiento.

\section{Historia de la Computación}

Se menciona que en los años 1960 empezaron a aparecer disciplinas relacionadas con la computación a nivel de estudios de pregrado [1], inicialmente con tres tipos de programas bien definidos en cuanto a su relación con la computación: ciencia de la computación, ingeniería eléctrica, y sistemas de información. Así, los estudiantes que querían ser expertos en el desarrollo de software o en sus aspectos teóricos, elegían la computación; aquellos que querían trabajar con hardware, elegían la ingeniería eléctrica; y quienes querían usar hardware y software para resolver problemas de negocios, elegían sistemas de información. Cada una de estas tres disciplinas tenía su propio dominio, y no se hablaba de que fueran parte de una familia de disciplinas informáticas. En aquella época fue la ingeniería del computador (computer engineering) la que más se desarrollaba pero como un área de especialización dentro de la ingeniería eléctrica.

Con el advenimiento del microprocesador a mediados de la década de 1970, la ingeniería del computador empezó a convertirse en una disciplina en sí misma, consolidándose en la década de 1990, al igual que la 
ciencia de la computación que fue aceptada como una disciplina académica con su propio cuerpo de conocimiento.

La ingeniería de software surgió como un área dentro de la ciencia de la computación por la dificultad de crear software confiable, cuando los programas llegaban a ser grandes y complejos. Así, la ingeniería de software se enfoca en métodos rigurosos para diseńarlos y construirlos de manera confiable y hagan lo que se supone que deben hacer.

Antes de la década de 1990, los sistemas de información se enfocaban principalmente en las necesidades informáticas clásicas del mundo empresarial: sistemas contables, sistemas de personal, sistemas de inventario, etc. Con la proliferación de las computadoras personales, los sistemas de información se hicieron más importantes en el tratamiento de problemas de gestión de información, así como en el uso apropiado de la información y la tecnología que da soporte a la eficiencia y efectividad organizacional.

La tecnología de la información surge a fines de la década de 1990, a consecuencia de la proliferación del uso extensivo de las computadoras que hacía cada vez más compleja el funcionamiento eficiente y disponible de la infraestructura informática de las organizaciones.

Después de los noventa estas áreas se consolidan, como una familia de disciplinas cada uno con su propio cuerpo de conocimiento, Ingenieria del computador (computer engineering) orientado al hardware y software del computador, ciencia de la computación e ingeniería de software orientado al estudio y desarrollo del software, y las tecnologías de información y los sistemas de información relacionados a las necesidades organizacionales.

\section{Disciplinas de la Computación}

En esta sección se describe brevemente cada una de las disciplinas mencionadas anteriormente.

Ingeniería del computador se interesa en el diseño y la construcción de computadoras y sistemas informáticos. Se centra en el estudio de hardware, software, comunicaciones y la interacción entre ellos. Se soporta en las teorías, principios y prácticas de la ingeniería electrónica y las matemáticas y los aplica a los problemas del diseño de computadoras y dispositivos basados en ellas.

Ciencia de la computación es un área base para el desarrollo de las otras áreas que abarca una amplia gama, desde sus fundamentos teóricos y algorítmicos hasta desarrollos de vanguardia en robótica, visión artificial, sistemas inteligentes, bioinformática y otras áreas apasionantes. Se extiende desde la teoría hasta la programación.

Sistemas de información enfatiza la integración de soluciones de tecnología de la información y procesos empresariales para satisfacer las necesidades de información de las organizaciones de manera efectiva y eficiente.

Tecnología de la información su énfasis está en la tecnología, seleccionando productos de hardware y software apropiado para una organización, integrando esos productos con las necesidades e infraestructura de la organización, e instalando, personalizando y manteniendo esas aplicaciones para los usuarios de organización.

La ingeniería de software es la disciplina del desarrollo y mantenimiento de sistemas de software que se comportan de manera confiable y eficiente, son asequibles para desarrollar y mantener, y satisfacen todos los requisitos que los clientes han definido para ellos.

\section{4. Áreas Temáticas de TI en el Perú}

En el ámbito nacional, en Julio del 2016, el Consejo Nacional de Ciencia y Tecnología e Innovación tecnológica, CONCYTEC [2] presenta el programa de nacional transversal de tecnologías de la información y comunicaciones (TIC); en ella define 4 áreas temáticas y 17 líneas de investigación prioritarias referido a las TIC, para hacer frente a los desafíos nacionales y a la necesidad de generar nuevos conocimientos de frontera e incrementar la capacidad tecnológica del país. Estas son:

4.1 Computación, orientada al desarrollo de la ciencia, tecnología e innovación en la teoría de la computación y diseño de sistemas computacionales en líneas como:

Interacción humano-computador, en donde según International Journal of Human-Computer Studies [3] los tópicos varían entre técnicas de interacción innovativas, interacción multimodal, interacción por habla, interacción gráfica, interacción lenguaje natural, interacción en móvil y sistemas embebidos, diseño de interfaces y evaluación de metodologías, diseño y evaluación de sistemas interactivos innovativos, prototipos de interfaz de usuario y sistemas de gestión.

Ingeniería de software, los tópicos [4] que comprende son : Métodos y herramientas a utilizarse en requisitos de software, diseño, arquitectura, verificación y validación, mantenimiento y evolución; métodos ágiles, dirigido por modelos, orientado a servicios, de código abierto y desarrollo de software global; enfoques para dispositivos móviles, multiprocesamiento, tiempo real, distribuido, basado en la nube, sistemas confiables y virtualizados; factores humanos y gestión del desarrollo de software; gestión de datos y problemáticas de big data de los sistemas de software; métricas y evaluación, minería de datos de recursos de desarrollo de software; aspectos económicos y de negocio del proceso de desarrollo de software.

Computación gráfica e imágenes describe la generación y manipulación de imágenes, permite la comunicación visual a través de la computación, sus aplicaciones son diversas, tales como animación, efectos 
especiales, video juego, imágenes médicas; esta área de conocimiento toca tópicos [5] como gráficos interactivos por computadora, visualización y modalidades de entrada novedosas, incluidos entornos virtuales, y, dentro de este alcance, modelos gráficos, estructuras de datos, lenguajes, algoritmos de manipulación de imágenes y software relacionado.

Computación ubicua, se refiere a crear inteligencia ambiental donde los dispositivos de red integrados en el entorno proporcionan conectividad y servicios discretos todo el tiempo, mejorando la experiencia humana y la calidad de vida sin una conciencia explícita de las comunicaciones subyacentes y las tecnologías informáticas. Según [6] los tópicos son: Penetrante/computación ubicua y arquitectura de comunicaciones y protocolos; computación autonómica; sistemas de computación en móvil y servicios; computación adaptativa, ambiental, invisible e implícita; la red móvil y la computación de igual a igual; paradigmas algorítmicos, modelos y análisis de sistemas de computación penetrante; espacios y entornos inteligentes; desarrollo de tecnologías (ej. Bluetooth, BANs, PANs, LANs inalámbricas); sistemas embebidos y computadoras portátiles; redes sensores inalámbricos, computación consciente, tecnología de agentes, del contexto, problemas de seguridad, privacidad, tolerancia a fallas y resiliencia, entre otros.

4.2 Sistemas cognitivos, según [2], se refiere a la integración de ideas, conceptos, constructos, teorías, modelos y técnicas de múltiples paradigmas, perspectivas y disciplinas en el análisis, comprensión y el diseño de los sistemas naturales o artificiales de procesamiento de información capaces de percepción, aprendizaje, razonamiento, comunicación, actuación y comportamiento adaptativo. Define líneas como:

Procesamiento digital de señales, en [7] los tópicos clásicos son: teoría, diseńo y algoritmos de tratamiento de señales digitales de audio, vídeo, imagen, comunicación, sonar, radar y médicos y tópicos emergentes tales como: big data, machine learning, internet de las cosas, seguridad de la información, biología de sistemas y biología computacional, vehículos autónomos, análisis de series temporales financieros, computación cuántica, ingeniería neuromórfica, interacción humano-computador e interface inteligentes de usuarios, procesamiento de señales del medio ambiente, procesamiento de señales geofísicas, química informática y bioinformática, gestión y prevención de desastres, energía renovable, entre otros.

Sistemas inteligentes, trata los tópicos [8] siguientes: inteligencia artificial, reconocimiento de patrones, redes neuronales, computación suave y sistemas difusos, visión por computadora y visión activa, Procesamiento y análisis de imagen y video, imágenes médicas, máquinas de aprendizaje, minería de datos, algoritmos evolutivos, inteligencia de enjambre, agentes inteligentes y multiagentes, ontologías, sistemas de soporte a la decisión, aprendizaje supervisado, semi supervisado y no supervisado, interacción humano computador y sistemas, gene- ración y reconocimiento de lenguaje, Modelos y Teorías Computacionales de la Cognición Humana, Robots inteligentes, representación y gestión del conocimiento, aprendizaje bayesiano, sistemas de interfaz cerebro-máquina, agrupamiento evolutivo, procesamiento de lenguaje natural, entre otros.

Neurociencias, las áreas de interés son el modelado, control y análisis de los sistemas neuronales en la neurociencia del comportamiento, neurociencia molecular y celular; neurociencia cognitiva, neurociencia del desarrollo, sistema autonómico y endocrino, neurología y sistemas sensoriales. [9]

Robótica y automatización, Sistemas automáticos de supervisión, control y gestión de procesos productivos, sistemas de control con inteligencia artificial utilizados en robots de todo tipo y servicio, y con énfasis en el aprendizaje y el control basado en sensores en el contexto de los sistemas autónomos. [10]

\subsection{Ciencia de datos}

Comprende los procesos y sistemas para extraer conocimiento o ideas a partir de datos en diversas formas, ya sean estructurados o no estructurados.

Comportamiento humano, Trata sobre el uso de las computadoras en la psicología, psiquiatría y disciplinas relacionadas, así como el impacto psicológico del uso de las computadoras en las personas y la sociedad; explorando además el uso de las computadoras en la práctica profesional, entrenamiento, investigación y desarrollo de teorías; los efectos psicológicos de las computadoras en el desarrollo, aprendizaje, cognición, personalidad humana y en las interacciones sociales, entre otras. [11]

Psicolingüísticas en TIC, estudia el proceso de comunicación, incluyendo el desarrollo del habla y traducción automática así como los problemas de significado lingüístico o semántico, desarrollo del lenguaje y el habla, así como el modelado computacional del lenguaje.[12]

Computación paralela distribuida, involucrada en la teoría, diseño, evaluación y uso de sistemas de computación distribuida y paralela, en áreas tales como: la implementación de algoritmos paralelos, arquitecturas de computadoras modernas, multiprocesadores con memoria compartida, sistemas de igual-a-igual, redes de sensores distribuidas, computación penetrante, computación óptica, lenguajes, compiladores y sistemas operativos, computación tolerante a fallas, bioinformática, ciberseguridad y ciber confianza, computación grid, entre otros. [13]

\subsection{Plataforma TIC}

Redes TIC, abarca las tecnologías de telecomunicaciones (ópticas, radiofrecuencia), redes de datos, protocolos e infraestructura de computación paralela y distribuida [2]. 
Internet de las cosas, en relación con arquitecturas IoT centradas en las cosas, centradas en los datos y centrada en los servicios; plataformas de sistemas ciberfísicos y SCADA, IoT basado en la nube, seguridad y gobernanza de sistemas IoT. También en tecnologías que hacen posible IoT tales como sensores, identificación por radio frecuencia, bajo consumo de energía, redes de sensores, sistemas en tiempo real, analítica de datos en IoT, procesamiento in situ, y software embebido. Además de los servicios, aplicaciones, estándares y bancos de prueba en IoT tales como plataformas de gestión de datos, audio y video, plataformas de minería, middleware de servicios, plataformas de servicio abierto, gestión de servicio semántico, protocolos de seguridad y privacidad, servicios y aplicaciones inteligentes, plataformas de soporte de aplicaciones IoT [14].

Redes de energía, relacionado más a la Ingeniería Eléctrica, comprende (1) las medidas de eficiencia energética, la reducción de la demanda de energía y la reducción de la demanda de energía de los servicios/movilidad; (2) redes de energía, incluyendo a las redes de pequeña y mediana generación [2].

Circuitos y sistemas electrónicos, relacionado más a la Ingeniería electrónica abarca los temas involucrados en el diseño electrónico con un nivel de abstracción adecuado; las alternativas de diseños existentes, identificando sus ventajas técnicas y/o económicas e incluyendo sus inconvenientes y otros factores de compromiso; y en las herramientas existentes, su uso y su influencia en la solución obtenida [2].

Ciberseguridad, comprende los tópicos de : Seguridad e integridad del servicio, prevención y detección de delitos informáticos, informática forense y seguridad, protección de confidencialidad, criptografía y protección de datos; seguridad de bases de datos y datos, protección de denegación de servicio; seguridad del comercio electrónico, vigilancia electrónica, fraude/ hackers /detección de terrorismo/ prevención, información, guerra de la información, seguridad nacional; ética de la información; cuestiones de privacidad de la información, sistemas de información / seguridad de la información, compartiendo; abuso por internet, prevención de intrusos en la red, seguridad de internet/red; código malicioso/protección de acceso no autorizado, seguridad de transacción, controles de virus / gusanos; gestión del riesgo, sistemas críticos para la seguridad; Tecnología de comunicaciones seguras y sistemas informáticos seguros; Medidas de control de seguridad, modelos de políticas y mecanismos; Arquitecturas de software y hardware; Seguridad de la red inalámbrica / móvil [15].

\section{Discusión}

En el desarrollo del área de la computación se fueron formalizando diversas disciplinas relacionadas con el uso de hardware y software, cada una con su respectivo cuerpo de conocimiento. En el Perú se creó la carrera de
Computación en la Universidad Nacional Mayor de San Marcos a fines de la década de 1960, sin embargo, en la actualidad, la formalización o normalización de estas disciplinas y cuerpos de conocimiento no existe o no se percibe con claridad, y de este modo han surgido una serie términos tales como ingeniería de sistemas, ingeniería informática, ingeniería de sistemas e informática, ingeniería industrial y de sistemas, computación científica, etc.

En las áreas de conocimiento determinadas por Concytec como impulsora del desarrollo nacional, se observa que la área denominada como ciencia de los datos, exhibe algunas líneas de interés específicas, sin embargo existen otras de bastante interés y que se están desarrollando en los últimos años, como: analítica de datos en general, big data, análisis de sentimientos (opinión), minería de datos, de textos, web y de procesos, que podrían incluirse mejorando el alcance de esta área temática.

En nuestra facultad, se consideró una línea más, denominada en forma general como "gestión y gobierno de TIC", dada la importancia de que los actuales gestores de TI, tengan las nociones tanto de las TIC como de la gestión mejorando a nivel táctico y estratégico la competitividad de las organizaciones.

Por otro lado se hace necesario mencionar que debido a los constantes retos inherentes a los avances de la tecnología de la información y comunicaciones, existe dos áreas que ACM propone podrían establecerse como disciplinas de la computación, éstas son: la ciberseguridad y la ciencia de los datos.

\section{Comentarios finales}

Las áreas y líneas de investigaciones vistas arriba son amplias, diversas, complejas, y se siguen incrementando y evolucionando cada vez más, incluso siguiendo la tendencia mundial de relacionarse fuertemente con otras disciplinas del conocimiento, de la ciencia y la ingeniería. Por otro lado las políticas de ciencia y tecnología en el país en estos últimos años ha promovido la investigación, con programas nacionales, financiamiento, becas, librerías digitales de journals internacionales de impacto y relaciones interinstitucionales y en nuestra universidad la formación de grupos de investigación podría propiciar una vasta serie de investigaciones que beneficien a nuestra sociedad.

En ese sentido invitamos a la comunidad académica a presentar sus trabajos de investigación en la Revista Peruana de Computación y Sistemas.

Los editores

Rosa Delgadillo Avila

Armando Fermín Pérez 


\section{Referencias bibliográficas}

[1] Shackelford, R., McGettrick, A., Sloan, R., Topi, H., Davies, G., Kamali, R., Cross, J., Impagliazzo, J., LeBlanc, R. and Lunt, B., Marzo 2006. "Computing curricula 2005: The overview report". https://www.acm.org/binaries/content/assets/ education/curricula-recommendations/cc2005-march06final. pdf, acceso 27 noviembre 2017.

[2] Programa Nacional Transversal de Tecnologías de la Información y Comunicación, CONCYTEC. https://portal.concytec.gob.pe/index.php/programas-nacionales-transversales-de-cti/programa-tic, acceso 20 octubre 2016.

[3] "International Journal of Human-Computer Studies". https://www.journals.elsevier.com/international-journal-of-human-computer-studies/, accesado 25 enero 2018.

[4] "Journal of Systems and Software". https://www.journals.elsevier.com/journal-of-systems-and-software/, accesado 14 octubre 2016.

[5] “Computers \& Graphics". https://www.journals.elsevier.com/ computers-and-graphics/, accesado 22 octubre 2016.

[6] "Pervasive and Mobile Computing". https://www.journals.elsevier.com/pervasive-and-mobile-computing/, accesado 15 enero 2018.

[7] "Digital Signal Processing". https://www.journals.elsevier. com/digital-signal-processing/, accesado 12 enero 2018.
[8] "Journal of Intelligent Systems". https://www.degruyter.com/ view/j/jisys, accesado 05 noviembre 2016.

[9] “Journal of Computational Neuroscience". http://www.springer.com/biomed/neuroscience/journal/10827, accesado 26 febrero 2018.

[10] "Robotics and Autonomous Systems". https://www.journals. elsevier.com/robotics-and-autonomous-systems, accesado 18 febrero 2017.

[11] "Computers in Human Behavior". https://www.journals.elsevier.com/computers-in-human-behavior, accesado 18 enero 2018.

[12] “Journal of Psycholinguistic Research". https://link.springer. com/journal/10936, accesado 25 febrero 2018.

[13] "Journal of Parallel and Distributed Computing". https://www. journals.elsevier.com/journal-of-parallel-and-distributed-computing, accesado 20 febrero 2018.

[14] "IEEE Internet of Things Journal". http://ieee-iotj.org/, accesado 22 febrero 2018.

[15] "International Journal of Information and Computer Security". http://www.inderscience.com/jhome.php?jcode=ijics, accesado 14 febrero 2018. 
